\title{
The Expression of the Follicle-stimulating Hormone Receptor in Spermatogenesis
}

\author{
Leslie L. Heckert* and Michael D. Griswold ${ }^{\dagger}$ \\ *Department of Molecular and Integrative Physiology, University of Kansas Medical Center, \\ Kansas City, Kansas 66160; 'School of Molecular Biosciences, Center for Reproductive Biology, \\ Washington State University, Pullman, Washington 99164-4660
}

\begin{abstract}
Results from experiments using mouse models suggest that the role of follicle-stimulating hormone (FSH) in spermatogenesis is the regulation of Sertoli cell proliferation and, ultimately, the size and spermatogenic capacity of the testis. The regulation of the expression of the FSH receptor (FSHR) gene is very cell specific and plays an initial role in the ultimate response of the Sertoli cells to FSH. The extreme cell specificity and the importance of the FSH response to spermatogenesis have led to an extensive characterization of the promoter of the FSHR gene. Several widely expressed transcription factors - including USF 1 and 2, GATA-1, and SF-1 and potential elements such as an E2F site and an Inr region - have been shown to contribute to the maximal transcription of the transfected FSHR gene. However, these experiments have failed to provide clues as to the cell-specific expression of the FSHR gene. In both cell transfections and in transgenic mice, the promoter can direct expression of transgenes promiscuously. The rodent FSHR promoter contains conserved $\mathrm{CpG}$ dinucleotides that were shown to be methylated in nonexpressing cells and tissue but unmethylated in Sertoli cells. The methylated $\mathrm{CpG}$ sites could interfere with the binding of general transcription factors and/or lead to a repressive chromatin structure in the nonexpressing cells. While yet-undiscovered cell-specific factors may play a role in the expression of the FSHR gene, repression and activation of local chromatin structure are likely to be involved.
\end{abstract}

\section{Biology of the Follicle-stimulating Hormone (FSH) Receptor in Spermatogenesis}

The primary hormonal controls on spermatogenesis involve the action of FSH and testosterone on Sertoli cells. Many of the studies examining the actions of FSH have been done on cultured Sertoli cells from 10- to 30-day-old rats. The Sertoli cells from rats of this age are easily placed in culture and respond to FSH with increased levels of cyclic AMP (cAMP), increased protein synthesis, and increased estradiol production (Fritz et al., 1976). As the age of the rat increases to 40 days or more, the response of Sertoli cells both in culture and in vivo changes. There is a large increase in the phosphodiesterase activity in the cells and the accumulation of cAMP and the subsequent stimulation of specific protein 
synthesis are curtailed (Means et al., 1976; Griswold, 1993). Responses to FSH in Sertoli cells from the adult rat are usually only measurable in the presence of a phophodiesterase inhibitor. In other words, at least in some rodents, the biochemical activities of FSH in the prepubertal animal appear to be assumed by testosterone in the adult. These findings have led to considerable controversy concerning the role of FSH in male reproduction (Zirkin et al., 1994).

In the past several years, some key experiments have clarified the overall biological role of FSH in rodent testes. First, Handelsman and colleagues administered testosterone alone to the gonadotropin-releasing hormone ( $\mathrm{GnRH})$ deficient mouse and showed that this treatment was sufficient for testicular maturation and fertility (Singh et al., 1995). GnRH-deficient mutant mice treated with testosterone implants had normal spermatogenesis but reduced testis size and germ cell numbers. Second, in a parallel study the same group showed that FSH treatment, in addition to testosterone treatment, resulted in GnRH-deficient mice with quantitatively normal spermatogenesis and testes of normal size (Singh and Handelsman, 1996). The exogenous FSH treatment increased testis size by $43 \%$. They concluded from these studies that FSH treatment during the first 2 weeks of life increased Sertoli cell numbers and total sperm production by the mouse testis. Third, it has been demonstrated that prepubertal treatment of normal rats with additional FSH produced larger-than-normal testes and higher total germ cell numbers (Meachem et al., 1996). Finally, several gene knockout experiments have contributed to this issue. A FSH $\beta$ gene knockout has resulted in a line of mice where the males are fertile but have smaller-than-normal testes and reduced germ cell numbers (Kumar et al., 1997). The FSH null mutants had testes that were about half normal size and, at 6-7 weeks of age, the number of epididymal sperm was reduced by $75 \%$. Two groups have produced FSH receptor null mice (FORKO) and have shown that while the males were fertile, the size of the testis and the total number of sperm produced were decreased significantly (Dierich et al., 1998; Abel et al., 2000). A FSH receptor null mutation $(566 \mathrm{C} \rightarrow \mathrm{T})$ has been reported in five male humans (Tapanainen et al., 1997). The men showed variable degrees of spermatogenic failure but were fertile. None of the five had normal sperm parameters and testicular size was reduced but two of the men had fathered two children each.

The use of the mice with the null mutations in the GnRH and FSH genes and the discovery of fertile men with inactivating mutations in the FSH receptor gene have shown clearly that FSH is not required for fertility in mice or men. However, these experiments also support the role for FSH in testis size and, ultimately, in spermatogenic capability. In addition, there is some evidence that, in the FORKO mice, the quality of the sperm produced is decreased (Krishnamurthy et al., 2000). The proliferation of Sertoli cells is maximal in 20- and 21-day-old rat fetuses and declines steadily until the second week after birth, when further cell division is rare (Orth, 1984). The role of FSH in the prenatal 
and newborn rat as a Sertoli cell mitogen is critical in the ultimate spermatogenic capability of the testis. When cytosine arabinoside, an inhibitor of DNA synthesis, was injected into testes of newborn rats, there was an overall $54 \%$ decrease in the size of the Sertoli cell population in the subsequent adults but the ratio of round spermatids to Sertoli cells was similar to controls (Orth et al., 1988). Thus, the Sertoli cell population was limiting to overall sperm production and underscored the importance of FSH in the establishment of the number of Sertoli cells and, therefore, the spermatogenic capacity of the adult (Orth et al., 1988).

\section{The FSH Receptor (FSHR) Gene}

The structure and organization of the FSHR gene was elucidated in both rats and humans, revealing that the receptor is encoded within 10 exons spanning at least $84 \mathrm{~kb}$ of DNA (Figure 1) (Heckert et al., 1992; Gromoll et al., 1996). The first nine exons encode the large extracellular amino-terminal domain of the receptor, while exon 10 encodes the transmembrane and intracellular portions of the protein. Exons 2 through 8 are both similar in length (68-77 bp) and amino acid sequence and, when aligned with each other, a similar repeated motif known as a leucine-rich repeat emerges from the sequences (Sprengel et al., 1990; Heckert et al., 1992). This motif, which is characterized by a pattern of commonly positioned aliphatic amino acids (leucine, isoleucine, valine, and phenylalanine), also was present in exon 9 but the sequence diverged from the patterns observed in exons 2-8. Typically, leucine-rich repeats are involved in cell-cell-specific adhesion and protein-protein interactions. With the FSH receptor, these repeated units are known to be involved in hormone binding and selectivity (Davy et al., 1977; Braun et al., 1991; Kobe and Deisendorfer, 1994). Interestingly, this repeated motif is present in other receptors within the larger superfamily of G protein-coupled receptors, of which FSHR is a member. This includes the other two glycoprotein hormone receptors, luteinizing hormone receptor (LHR) and thyroid stimulating hormone receptor (TSHR), and several more recently described receptors. Together, they make up the growing family of leucine-rich-repeat-containing, G protein-coupled receptors (LGRs) (Loosfelt et al., 1989; McFarland et al., 1989; Parmentiar, 1989; Akamizu et al., 1990; Hsu et al., 1998,2000).

Phylogenetic analysis of members within the LGR family identified three subgroups in which one subgroup is made up of the glycoprotein hormone receptors and the LRG receptors identified in sea anemone, Caenorhabditis elegans, and Drosophila (Hsu et al., 2000). When the gene structures are compared between family members, some striking similarities are unveiled, indicating that a receptor gene identified in cnidarians (e.g., sea anemones, corals, Hydra), the lowest animal group having a nervous system, and the mammalian glycoprotein receptor genes are evolutionarily linked (Figure 1) (Vibede et al., 


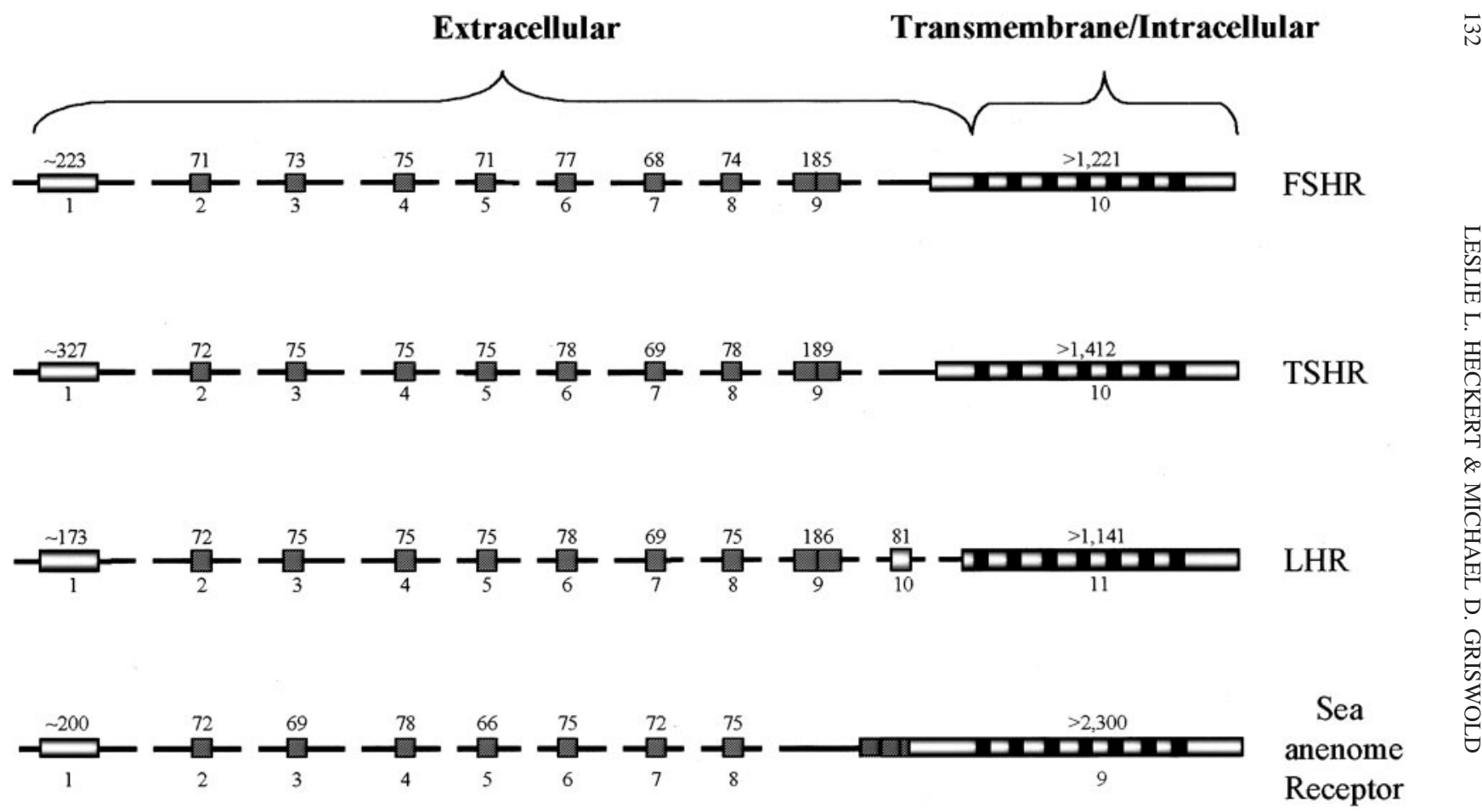

FIG. 1. Comparison of the gene structures for the glycoprotein hormone receptors and sea anenome receptor. Exons are depicted as boxes with their number given below and their size in bp given above. Hatched boxes represent leucine-rich repeats and the black boxes, the seven membrane-spanning domains. For the glycoprotein hormone receptor genes, the structures were derived from the rat FSHR (Heckert et al., 1992), human TSHR (Gross et al., 1991), and rat LHR (Koo et al., 1991; Tsai-Morris et al., 1991). The gene structure for the receptor from sea anemone was derived from studies by Vibede et al. (1998) (Accession nos. AF084384-AF084390). 
1998). While small differences in the number of exons exist among these receptors, the general structure follows that described for FSHR, whereby the leucine-rich motifs within the amino-terminal domain are encoded by multiple small exons and the seven membrane-spanning, C-terminal intracellular domain is encoded by the last and largest exon (Gross et al., 1991; Koo et al., 1991; Tsai-Morris et al., 1991; Vibede et al., 1998). Of note, the phasing of the exons within each of the genes is identical (i.e., they are all in phase 2). Thus, it appears that the FSHR, TSHR, and LHR genes share a common ancestor that arose prior to cnidarian evolution (Vibede et al., 1998).

\section{Transcriptional Regulation of FSHR}

\section{A. TRANSCRIPTION FACTORS AND TRANSFECTIONS}

The expression of FSHR is remarkably cell specific. It is found in testicular Sertoli cells and ovarian granulosa cells, where receptor levels help determine the physiological response to FSH by communicating the pituitary-derived FSH signal to these cells in the gonads. The mechanisms that regulate FSHR gene transcription are of interest because they not only provide insight into endocrine regulation but also into cell-specific gene expression. Most of the data concerning the expression of the FSHR gene were generated by evaluation of its promoter in transient transfection assays and transgenic mice. While progress has been made regarding the mechanisms that control basal transcription and regulation by the hormone FSH, the mechanisms that drive cell-specific expression remain elusive.

Studies on the rat gene have provided the vast majority of information on FSHR transcription and thus, for simplicity, we will review findings on the rat promoter and add important detail and support from the other species where appropriate. Alignment of the DNA sequences of the FSHR promoter regions from rat, mouse, human, and ovine revealed a high degree of sequence conservation spanning from the translational start codon to approximately $1050 \mathrm{bp}$ upstream (Figure 2) (Huhtaniemi et al., 1992; Gromoll et al., 1994; Sairam and Subbarayan, 1997; Heckert et al., 1998). Thereafter, the sequence similarity drops significantly. In the rat gene, transcriptional start sites were mapped to positions -80 and -98 relative to the translational start codon (Heckert et al., 1998) (Figure 2). These sites corresponded well to those identified for the human gene but differed significantly from those originally reported for the mouse and ovine genes, which were located at positions $-534 \mathrm{bp}$ and $-163 \mathrm{bp}$, respectively (Figure 2) (Huhtaniemi et al., 1992; Gromoll et al., 1994; Sairam and Subbarayan, 1997). Examination of the sequences within the proximal promoters revealed that the FSHR gene is a member of a class of genes whose promoters lack a canonical TATA motif. Different classes of TATA-less promoters have been described and include the housekeeping genes, which are a groups of genes 
†ouse (-534)

rat -513 A-TATCAGATGGGACACAGGAAAACAGCAACACCAAATACT----AAAGGTAAATTTTGT mouse -543 A-TAACAGATAGGTCACAGGAAAACAGCAATGCAAAATACT----AAAGGTAAATTTTGT human -453 TCTCTCAGGTGGGCTGGTTGAAGAAATTGATGCTGAAGACT----GAAGGTCCCAG---ovine -511 TGPATCAAGCAGCCTGGAGGAMGACATTGACACC-AAGACTGGAAACAGGTCCCTG----

rat -458 TCCCTTCACATCAOTCGT-------TAGAGATGTGTCATGT----GGATGTG-CTTG-mouse -488 CCCCTTCATGTCAGTAGTACA-----TTAGAGATGTGTCATAT----GGATGTA-CTTGCC human -401 -CCCTTCAC-TTATVAGTACCCCTCTTAGTGATGTGTCATATTGTGGGTTOTGTCTTTTT ovine -456 -ACCTTCAC-TGAGGAACTC---TCTTPATGATOTTTCACACTGCAGATTGCATCTGTTT

rat -413 GGGAGAGGGTCAA-----TTATGTCACTGAGG----AG---AAGACAGTAGTGACCAGTAG mouse -437 GGGA-AGGGACGAACCCTGATACACA human -343 TGGAGAAAGCAA----TCATGTCACTCTGTT--GAG---AaGAGAATGGTGAACAGCAA OVine -401 TGGAGAAAOTCAA----GCGTGTCACTCTETTTTGAGAGAAAAAAAATAGTGACCCACAG rat -364 GGACCCCTGTGCAGCAAAAT-AATGTGAATCTGCTGCTAT---------AGACTGATATC mouse -388 G-ACCTCCATGCAGTAAAAT-AATGTGAATCTGCTGATATCAGTCCATTAGACTGATATC

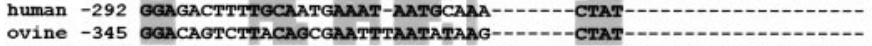

rat -314 TTCAAATATGCACCAAGTTTCTCTTYTCTGTCATTTTGGGGGTCAAGGAATAAAAAATAT mouse -330 TCTAAATATGCACCAAGTTTCTACTTGTTGTCATTTTGG-GGTCAAGGAATAGAAAATAT human -260 TCCAGACATGCCTAATGGTTCTATTTGCTGTGTGCCTTA-GGTCAGGGTGTAAGAAACCC ovine -312 TCTAGACATGCATCAAGTTTCAATTTGCAAAC---------CCAACC---AAAAAAGGT

AP-1

rat -254 AGGTCTTGAAGGATAAAGCAGAAGATtATTGACACACATTAGTCACAT---:------mouse -271 AGGTCTTGAAGGATAAGACAGGTGCTTATTGACAAATATTAATCACATTTCAATCATGTA human -201 AA-TCTTGAAGGAAAACAGAGTAGCTTAT---
ovine -265 A---AAGG--ACAGCGTATCTTCCACGCCCTCTACCTCTCCCACCCCACCCCCA $\longleftrightarrow$ human $(-184)$

rat -205 TTAATATATATAATCACTATTGACACATATTAATTrTACT---TGCCTGGAAGC̈GAC--mouse -211 TTAATACATATAGTTACTACGGACACATATTAATTTTACT---TGCCTGGAAGCGAC--human -173 -.--_ovine -214 cCAA----AGTCACTGCTGTCACTCAGAAATTCTGCTATTTGTCTGGAAGTGACCGA
ovine $(-163)$

E box

rat -151 -TAA-----AAAAAA---AGGCATCTCTTGGTGGGTCAC̈GTGACTTTGCCC̈GTTCTCCA mouse -157 -AAAAGAAAAAAAAA---AAGCATCCTTTAGTGGGTCACGTGACTTTGCT-GTCCTCCA

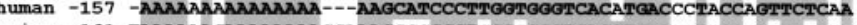
ovine -161 TAAAAAAGAAAAAAAAGGAAGCGGCCCTGGGCGGTCACGTGACCCTACCAGCTCCCAA

$$
\text { Inr GATA }
$$

E.2F rat -101 AGCA-GATCTCP-CTPATCCEGACAGTGTGTGGAGGAGCCTGGGGATCTGTGGAAGTTY mouse -102 AGCA-GATCTCT-CTTATCCGGACAGTGTGTGGAGGAGCCTGGGGAATCCGTGGAGGTTP human -101 GTCA-GATCTCTTCTCATAAGGGCACTETGTGGAG---CTTCTGAGATCTGTGGAGGTTT ovine -101 CGCAAGACCTCTTCTCAAAAGGGCTCAGTGTGGAG---CCTCTGAAATCTGGGCAGGATT $\longrightarrow$ rat $(-98) \underset{\text { human }(-99)}{\longrightarrow} \longrightarrow$ humat $(-89)$

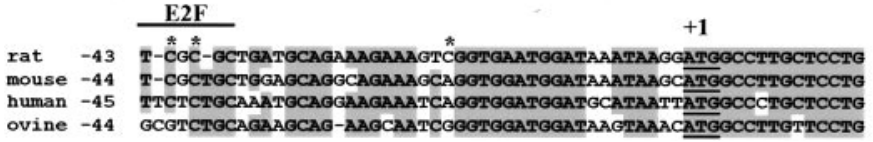

FIG. 2. Sequence comparison of the rat, mouse, human, and ovine FSHR promoter regions. Aligned sequences of the FSHR promoter regions from rat, mouse, human, and ovine, including the translational start site $(+1)$ and approximately $500 \mathrm{bp}$ upstream. Base positions are indicated in the left margin and are relative to the translational start codon (ATG underlined), where adenosine is represented as +1 . Arrows indicate transcriptional start sites identified in each species. DNA elements revealed as important for FSHR regulation are marked with a line over top of the sequence (E2F, Inr, GATA, E box, and AP1 sites). Asterisks mark cytosines within the rat FSHR promoter that were differentially methylated in nonexpressing and expressing cell types. 
that are constitutively active and ubiquitously expressed, and genes that are more restricted in their developmental and tissue-specific expression patterns. The FSHR gene more closely resembles the latter. Like the FSHR, genes that lack a TATA motif often initiate transcription from multiple sites and employ an initiator element (Inr) that assists in the positioning of RNA polymerase II (Smale and Baltimore, 1989; Weis and Reinberg, 1992).

Characterization of the FSHR promoter by transient transfection analysis has provided important insight into basal promoter function but revealed little with regards to the mechanisms responsible for cell-specific expression (Linder et al., 1994; Goetz et al., 1996; Heckert et al., 1998). Promoter evaluation in various cell types, both FSHR expressing and nonexpressing, showed that the promoter activity did not correlate with the cell's ability to express the endogenous FSHR gene. Thus, in primary cultures of Sertoli cells, where the endogenous receptor is actively expressed, promoter activity was only modestly higher than in some nonexpressing cell types but lower than in others (i.e., the MA-10 Leydig cell line). Thus, transient transfection analysis has failed to provide any clear-cut evidence that the $5^{\prime}$ flanking region of the rat gene imparts cell-specific expression. In part, this appears to be upheld in transgenic mice (see below).

Deletion and block-replacement mutagenesis have identified several important response elements within the FSHR promoter region (Linder et al., 1994; Goetz et al., 1996; Heckert et al., 1998). Promoters of various lengths have been included in the studies on FSHR transcription, with the largest fragment spanning out $5000 \mathrm{bp} 5^{\prime}$ to the start of translation (Linder et al., 1994; Heckert et al., 1998). Promoter deletion studies showed that most, if not all, of the positive regulatory elements participating in basal promoter function reside within the first $200 \mathrm{bp}$ of the promoter and suggested that repressor elements also contribute to control of FSHR transcription. Accordingly, the FSHR promoter could be deleted to approximately $200 \mathrm{bp}$ upstream of the translational start site without adversely impacting promoter activity when assayed in either primary cultures of Sertoli cells or the mouse Sertoli cell line, MSC-1 (Linder et al., 1994; Goetz et al., 1996; Heckert et al., 1998). In fact, promoter activity increased with some deletions, suggesting that transcriptional repressors bind elements located between -5000 and -2700 and between -2700 and -200 bp. Similar transcriptional increases were observed when the human and ovine promoters were truncated to within a few hundred bp of the translational start site (Gromoll et al., 1994). No further characterization of the potential repressor elements has been reported.

Elements that have been identified in the regulation of FSHR include an E box, AP-1 site, Inr, E2F site, GATA site, and two steroidogenic factor-1 (SF-1)-like binding sites (Figures 2 and 3) (Goetz et al., 1996; Heckert et al., 1998; Kim and Griswold, 2001; Levallet et al., 2001). Extensive block replacement analysis performed on the first $200 \mathrm{bp}$ of the promoter revealed the E box, 


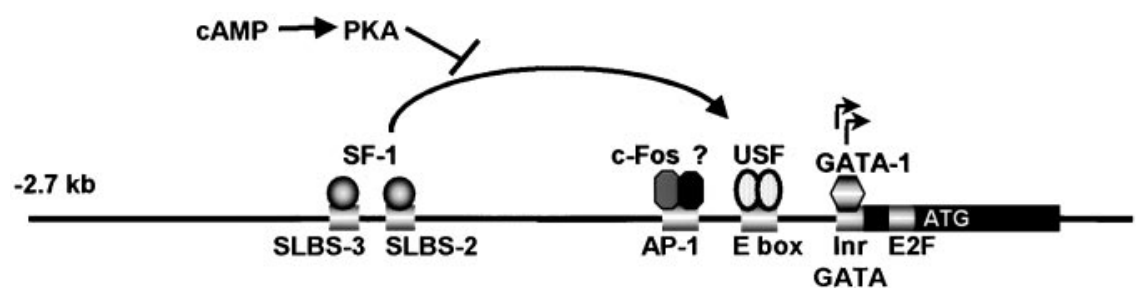

FIG. 3. Model for regulation of the FSHR promoter. Exon 1 of the FSHR gene is indicated as a black rectangle; important regulatory elements are shown by shaded white rectangles. Straight arrows indicate the transcriptional start sites. Regulatory elements, indicated below the depicted promoter, are the Inr, GATA, and E2F sites, located within exon 1, and the E box, AP-1 site, and two SF-1-like binding sites, SLBS-3 and SLBS-2, located within the promoter region. GATA-1 binds the GATA site, the upstream factor (USF) proteins (USF1 and USF2) bind and activate transcription through the E box, while SF1 induces FSHR transcription through the SLBS-2 and SLBS-3 elements. Following binding to SLBS-2 and SLBS-3, SF-1 is postulated to interact either directly or indirectly with the USF proteins, resulting in transcriptional stimulation. Activation of protein kinase A (PKA) by cAMP interferes with the ability of SF1 to transactivate the FSHR promoter. In the absence of the upstream SF-1 binding sites, cAMP stimulation results in the induction of cfos-containing complexes that bind the AP-1 site and stimulate FSHR promoter activity.

Inr, and E2F sites (Goetz et al., 1996; Heckert et al., 1998). A mutation through the $\mathrm{E}$ box had the single greatest impact on rat FSHR promoter activity, while more modest effects were observed with mutations in the E2F and Inr sites. In the rat gene, the E box $\left(5^{\prime}\right.$-CACGTG- $\left.3^{\prime}\right)$ is located at position -120 and shows a high degree of sequence conservation with the other species. Except for the human gene, which has a single G-to-A change at the first guanosine of the core sequence (5'-CACGTG-3' to $5^{\prime}$-CACATG-3'), each of the core bases is conserved across species (Figure 2). A requirement for the $\mathrm{E}$ box also was shown in studies with the ovine promoter (Xing and Sairam, 2001). Mutagenesis of both the core and flanking sequence of the E box revealed that a change in the core's central CG dinucleotide (CACGTG) to either GC or GA diminished promoter activity $60-80 \%$ but was tolerated to a greater extent than a change in the core's flanking bases (5'-GGTCACGTGACTT-3' to 5'-GtaCACGTGtaTT-3') (Heckert et al., 1998). These studies also showed that the sequence difference within the human E box had little impact on promoter function (Figure 2). Furthermore, in vivo genomic footprinting revealed that the $\mathrm{E}$ box within the endogenous FSHR gene is bound by proteins but only in cells that expressed the receptor (Heckert et al., 2000).

DNA/protein binding analysis identified the ubiquitously expressed transcription factors USF1 and USF2 as the predominant proteins binding the E box in Sertoli cells and showed that the bases required for binding to the element mirrored those needed for promoter activity (Figure 3) (Goetz et al., 1996; Heckert et al., 1998). Direct functional support for the USF proteins in transcrip- 
tional regulation of FSHR was obtained when co-transfection of wild-type or mutant forms of the USF proteins with FSHR reporter constructs confirmed that the USF proteins activated the FSHR promoter by binding the proximal E box (Heckert et al., 2000). Furthermore, the first 40 amino acids of the USF proteins, as well as regions encoded by exon 5 and the USF-specific region (USR), were necessary for FSHR transcription. The observations that ubiquitously expressed USF proteins regulate transcription through the $\mathrm{E}$ box and that the $\mathrm{E}$ box of the endogenous FSHR gene is occupied by proteins only in cells that express the receptor suggest that the chromatin structure in expressing cells somehow allows access of the USF proteins to the $\mathrm{E}$ box, while, in nonexpressing cells, the structure is different and nonpermissive for USF binding.

Like the E box, the E2F, GATA, and Inr sites were implicated in the regulation of endogenous FSHR gene activity by in vivo genomic footprint analysis (Kim and Griswold, 2001). In addition, these elements have been examined on the FSHR promoter by mutagenesis and binding analysis but not as extensively as the E box. In addition to the footprint analysis, the E2F site (5'-TTTTCGCGC-3'), located from -38 to -46 , was implicated by a blockreplacement mutation that encompassed the entire site as well as by more refined mutations that specifically changed bases within the conserved element (Heckert et al., 1998). Examination of the sequence of this element revealed that it is significantly less conserved across species than the E box (Figure 2). The largest impact of this element was observed with a mutation within the second GC pair (underlined above), which decreased promoter activity to the same degree $(\approx 50 \%)$ as the full block-replacement mutant (Heckert et al., 1998). Interestingly, the position of this dinucleotide is conserved in each of the four species (Figure 2). While specific protein complexes were shown to bind the E2F site, they do not appear to contain the E2F1 protein, as antibodies against this factor failed to produce a supershifted complex (Griswold and Kim, 2001; Kim and Griswold, 2001).

A role for the Inr was determined from analysis of 3' deletion mutants and specific mutations within the element (Goetz et al., 1996; Heckert et al., 1998). This region of the promoter encompasses both transcriptional start sites, spanning from -103 to -80 . Specific protein complexes were shown to bind this region of the promoter and were competed by the well-characterized Inr from the deoxynucleotidyltransferase gene (Goetz et al., 1996). Interestingly, mutation of a GATA site located within this region significantly diminished FSHR promoter activity, suggesting that a member of the GATA family of transcription factors is important for FSHR promoter function (Kim and Griswold, 2001). In nuclear extracts from Sertoli cells, the GATA-1 transcription factor was found to bind this element, further supporting this suggestion. While GATA-1 binds within the Inr region of the promoter, several other proteins that interact with this element 
remain unidentified and may also contribute to FSHR transcription (Kim and Griswold, 2001).

Recently, studies on both the rat and mouse FSHR promoters demonstrated that the orphan nuclear receptor SF-1 regulates transcription of the FSHR gene. SF-1 is a key regulator of endocrine function and sex determination. Its expression is limited primarily to cells of the gonads, adrenal, pituitary, and ventral medial hypothalamus, where it is thought to contribute to cell-specific properties of genes expressed within these tissues (Hatano et al., 1994; Ikeda et al., 1994; Ingraham et al., 1994; Shen et al., 1994; Morohashi et al., 1995; Roselli et al., 1997). SF-1 is known to regulate transcription of several key steroidogenic enzymes as well as genes involved in the production of the gonadotropin hormones, including the luteinizing hormone (LH) $\beta$ subunit, the GnRH receptor, and the inhibin $\alpha$ subunit (Rice et al., 1991; Morohashi et al., 1992; Barnhart and Mellon, 1994; Asa et al., 1996; Keri and Nilson, 1996; Caron et al., 1997; Duval et al., 1997; Halvorson et al., 1998,1999; Dorn et al., 1999; Ngan et al., 1999; Tremblay and Drouin, 1999; Wolfe, 1999; Wolfe and Call, 1999; Ito et al., 2000). Identification of FSHR as a target gene for SF-1 regulation revealed yet another level in which SF-1 acts to control endocrine homeostasis.

Transfection studies with both the mouse and rat FSHR promoters showed that transcriptional activity increased substantially when co-transfected with an expression vector for SF-1 (Heckert, 2001; Levallet et al., 2001). In the mouse promoter, two SF-1-like binding sites (SLBS-2 and SLBS-3) (Figure 3) were required for response to the orphan receptor. In the rat promoter, SF-1 response was mapped to several regions upstream of -743 and within the first 200 bases of the promoter. Within the latter region, the $\mathrm{E}$ box was shown to be critical for response to SF-1, not only for the proximal promoter but also for response generated through the upstream site(s). In the absence of SF-1 binding to the $\mathrm{E}$ box, a role for the USF proteins in integrating the SF-1 response was implicated and subsequently substantiated through co-transfection studies with mutant USF proteins (Heckert, 2001). Notably, the requirement for USF in response to SF-1 was unique to the FSHR promoter, as USF levels in the cell did not influence SF-1 activation of the equine $\mathrm{LH} \beta$ promoter. Unlike the mouse promoter, upstream SF-1 binding sites were not identified in the rat gene but analysis of a series of truncated promoters suggested that multiple response elements were required in the upstream region.

Although the mechanisms by which SF-1 and USF cooperate to regulate FSHR are not fully understood, the finding that these transcription factors act in concert to activate transcription provides important insight into FSHR gene regulation, the transactivation function of SF-1, and the expanding physiological roles of SF-1 in the hypothalamic-pituitary-gonadal axis. Within this axis, SF-1 appears to regulate both FSH production and response. Thus, SF-1 can directly influence FSH production in the pituitary through its regulation of the $\alpha$ subunit 
of the FSH regulatory protein inhibin. SF-1 can also influence FSH response in the testis through its regulation of FSHR. Interestingly, activation of the inhibin $\alpha$ subunit by SF-1 has been shown to dramatically increase in the presence of activated protein kinase A (PKA), the major downstream effector of FSH action in the gonads (Ito et al., 2000). Thus, increasing levels of FSH in the presence of SF-1 should dramatically stimulate inhibin levels produced by the gonads, which would then feed back on the pituitary to specifically decrease FSH production. Remarkably, studies with the rat promoter revealed that activated PKA blocked SF-1-stimulated transcription of the FSHR promoter (Heckert, 2001). Thus, with SF-1 poised to regulate these two genes, FSH stimulation can lead to opposite transcriptional effects, resulting in a rise in $\alpha$ inhibin and a fall in FSHR, a result that would dramatically decrease FSH signaling in the gonads.

\section{B. REGULATION BY CAMP}

In males, FSHR mRNA levels are significantly reduced in response to FSH or agents that stimulate the cAMP pathway (Themmen et al., 1991; Maguire et al., 1997; Griswold et al., 2001). While earlier studies have suggested that post-transcriptional mechanisms are responsible, two more recent experiments implicate the involvement of transcriptional events (Maguire et al., 1997; Griswold et al., 2001). These latter studies showed that the transcriptional inhibitor actinomycin D blocked receptor mRNA downregulation and that FSHR hnRNA decreased in the presence of FSH (Maguire et al., 1997; Griswold et al., 2001). However, an FSHR promoter construct containing sequence from -383 bp to -1 bp was not negatively regulated by FSH treatment (Griswold et al., 2001). In fact, promoter activity increased approximately three-fold in the presence of FSH, an event that required an AP-1 site located at position - 213 in the promoter (Figures 2 and 3). The AP-1 element also was shown to specifically bind FSH-induced protein complexes that contained the transcription factor c-fos. While these studies did not resolve the mechanism by which FSHR transcription is diminished by FSH, they did appear to rule out a role for the transcriptional repressor inducible cAMP early repressor (ICER), which is also known to bind the AP-1 site (Monaco et al., 1995; Griswold et al., 2001). As mentioned above, a mechanism for the inhibitory effects of the cAMP pathway on FSHR transcription was suggested recently by the observation that the SF-1 activation of the FSHR promoter was blocked when this pathway was stimulated (Heckert, 2001). Thus, under conditions where SF-1 is activating the gene, stimulation of the cells with FSH would result in decreased transcription.

\section{TRANSGENIC MICE}

Studies in transgenic mice have been used to help delineate the region of the FSHR gene needed for cell-specific expression. In two separate studies, $5000 \mathrm{bp}$ 
of the rat FSHR promoter were used to drive expression of a reporter gene, either $\beta$-galactosidase or Cre recombinase, in transgenic mice (Linder et al., 1994; Heckert et al., 2000). In the first study, $\beta$-galactosidase expression was measured in various tissues by Northern blot analysis to help assess cell-specific expression (Linder et al., 1994). In two independent transgenic lines, evaluation of liver, thyroid, kidney, ovary, and epididymal RNA samples revealed that only testis and ovary expressed the transgene. While the cell types expressing the transgene were not determined, the results indicated that tissue-specific expression is directed by the first $5000 \mathrm{bp}$ of FSHR 5' flanking sequence, indirectly suggesting that cell specificity is directed by this portion of the gene as well.

In the second study, Cre recombinase expression was evaluated by reverse transcriptase polymerase chain reaction (RT-PCR) in RNA samples collected from testis, ovary, heart, lung, liver, kidney, brain, bladder, stomach, spleen, and eyes (Heckert et al., 2000). A second transgene containing only $198 \mathrm{bp}$ of 5' flanking sequence also was examined in this study. Of eight transgenic lines examined, the $5000 \mathrm{bp}$ Cre construct expressed in both testis and brain. Three of these lines also had marked expression in other tissues. Further examination revealed that testis expression of the transgene did not follow the same temporal pattern as that of the endogenous FSHR gene in that it was not expressed at postnatal day 10 . Interestingly, the smaller -198 Cre transgene appeared to better restrict expression, as three transgenic lines only expressed Cre recombinase in the testis. However, like the 5000 bp Cre construct, no expression was observed at postnatal day 10, despite expression of the endogenous FSHR gene. Closer inspection of two of the -198 bp Cre lines revealed that testis expression in mice either 27 or 50 days of age was mostly, if not entirely, due to inappropriate expression in germ cells. Thus, the inaccurate temporal expression of the transgenes and their expression in germ cells led to the conclusion that the promoter sequences did not contain enough information to properly restrict or express the receptor to Sertoli cells of the testis. It is important to note, however, that examination of the cell types expressing the transgene was limited to studies on the smaller construct, leaving open the possibility that Cre expressed from the $5000 \mathrm{bp}$ promoter occurred within the Sertoli cells but later than that observed for the endogenous gene. If true, this would indicate that different mechanisms control early (embryonic/early postnatal) and late (postpubertal) expression of the FSHR gene.

Studies on the luteinizing hormone receptor (LHR), a gene evolutionarily linked to FSHR, provided support for this theory, as they implicated different transcriptional mechanisms for early and late LHR expression (Hamalainen et al., 1999). Transgenic mice carrying $2 \mathrm{~kb}$ of the murine LHR promoter exhibited remarkable similarities to the FSHR transgenic mice. Thus, three out of five LHR transgenic lines exhibited expression within the testis but failed to express in the ovary, while all lines showed ectopic expression in the brain. Also of note was 
the finding that testis expression of the LHR transgene was not observed between weeks 1 and 3 after birth but appeared at 5 weeks of age in both the germ cells and Leydig cells. Because the transgene did not follow the temporal expression of the endogenous gene, separate transcriptional mechanisms for early and late expression were implicated. Further evaluation of the FSHR transgenes is necessary to determine if this is true for FSHR too. Thus, while it is currently unclear if elements sufficient for adult Sertoli cell expression of FSHR reside in the 5000-bp promoter, we can conclude from these studies that the evaluated promoters lack sufficient information to direct Sertoli cell expression in early postnatal testis as well as the information needed to prevent expression outside of this cell type.

\section{Role of Methylation and Chromatin Structure in FSHR Expression}

As described above, the region of the core promoter between -383 and +1 bp relative to the translational start site contains two Inr-mediated transcriptional start sites, an E box, and several other sites involved in transcriptional regulation of this gene. No cell-specific DNA-protein complexes have been found in the FSHR promoter that could mitigate the extreme cell specificity of transcription (Heckert et al., 1992,1998; Linder et al., 1994; Goetz et al., 1996). Reporter gene assays driven by the rat FSHR promoter have shown that this region could promote the promiscuous transcription of the gene in several cell lines in which the expression of the endogenous FSHR gene was originally repressed (Linder et al., 1994).

In mammals, DNA methylation patterns appear to play a critical role in terms of gene regulation in differentiating and differentiated cells (el-Deiry et al., 1991; Tate and Bird, 1993; Eden and Cedar, 1994). There is considerable evidence that a variety of cell-specific genes have distinctive DNA methylation patterns in expressing and nonexpressing cells, and undergo demethylation at the stage of gene activation (el-Deiry et al., 1991; Eden and Cedar, 1994; Beard et al., 1995; Nickel et al., 1995). The core promoter of the rat FSHR gene does not contain $\mathrm{CpG}$ islands but does contain a few specific $\mathrm{CpG}$ dinucleotides that are potential methylation sites (Heckert et al., 1992). Using bisulfite-based DNA sequencing, seven specific CpG sites within the FSHR core promoter were shown to be methylated in cells that did not express the gene and unmethylated in Sertoli cells (Figure 2) (McGuinness et al., 1994; Griswold and Kim, 2001).

In addition, the mouse Sertoli cell line (MSC-1) derived from a testicular tumor has an inactive FSHR promoter (McGuinness et al., 1994) and the inactive state of transcription in MSC-1 cells correlated with the cytosine methylation of the core promoter of the FSHR gene (Griswold and Kim, 2001). Treatment of MSC-1 cells with 5-azacytidine (5-azaCdR) resulted in the demethylation of the 
mouse FSHR promoter and reactivated the transcription of the FSHR gene. The drug 5-azaCdR is known to block DNA methylation in newly replicated DNA molecules and has been used to activate silent genes in cells whose expression of that gene is controlled by DNA methylation (Ottaviano et al., 1994; Bender et al., 1998; Bovenzi et al., 1999).

DNA methylation in the proximal promoter could potentially lead to regulation of the expression of the FSHR gene by directly interfering with or changing protein-DNA interactions at those sites. This changed interaction could lead to changes in transcription factor-DNA interactions or altered chromatin structure. Within the 320-bp region of the rat FSHR core promoter, four of the seven potential 5-methylcytosine residues are located within known protein-DNA binding sites (Figure 2). The methyl group(s) within cis-acting regulatory elements could interrupt the binding of trans-acting factors to their target sites on the promoter. For example, DNA methylation of the E box element (CACGTG, -124/-119) in the FSHR promoter region inhibited the binding of nuclear extracts from Sertoli cells by 3.5 -fold (Griswold and Kim, 2001). It also is clear that cytosine methylation can exert inhibitory effects through altering chromatin structure and not by inhibition of the transcription machinery directly (Tate and Bird, 1993; Richardson and Yung, 1999). A repressive chromatin structure can result from the recruitment of histone deacetylases (HDACs) by methylated cytosine binding protein (MeCP2) in nucleosomal DNA (Jones et al., 1998; Nan et al., 1998). It is possible that the FSHR promoter is an example of a gene where $\mathrm{CpG}$ methylation both inhibits transcription factor binding directly and promotes the formation of a repressive chromatin structure.

The chromatin structure surrounding the proximal promoter in the rat FSHR receptor has been examined in nucleosome reconstitution experiments (M.D. Griswold, unpublished data). The proximal promoter was reconstituted into nucleosomes using $319 \mathrm{bp}$ and chicken erythrocyte histones. The resulting nucleosomal locations were then mapped by hydroxyl radical footprinting. Examination of the sequence in the core promoter reveals the presence of possible strong nucleosome positioning sequences just $5^{\prime}$ of the $\mathrm{E}$ box. The hydroxyl radical footprinting showed that one nucleosome positioned very tightly over the region of the promoter from approximately -200 to $-400 \mathrm{bp}$. The second nucleosome positioned very weakly over the region from -100 to +100 and the $E$ box appeared to be localized to the linker region. The seven $\mathrm{CpG}$ methylation sites were positioned mainly within the second nucleosome but results of reconstitution experiments with promoter DNA where the $\mathrm{CpG}$ sites were methylated were unchanged from the first experiment. These results lead to a model (Figure 4) whereby the tightly positioned nucleosome helps to define the promoter site. The repressed state of the promoter could be achieved when the methylated DNA binds 


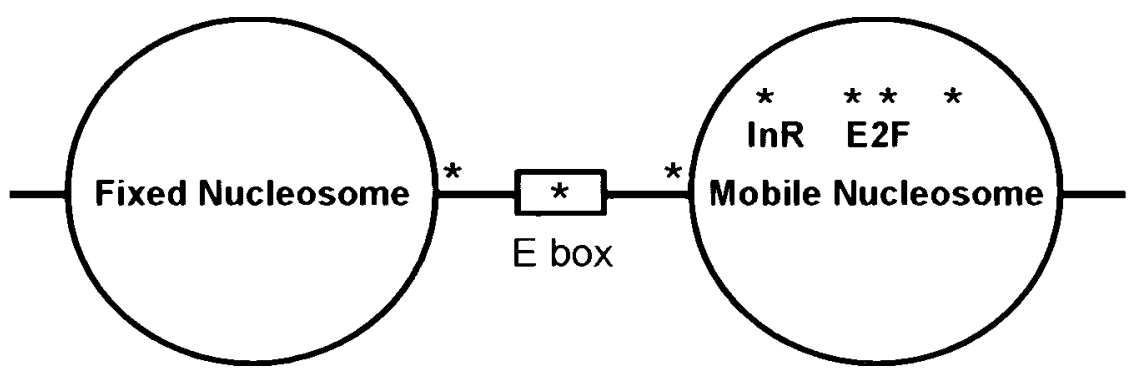

FIG. 4. Positioning of nucleosomes over the proximal FSHR promoter. Nucleosomes were reconstituted using 319 base pairs of the proximal FSHR promoter and chicken erythrocyte histones (Reeves and Wolffe, 1996). The positions of the reconstituted nucleosomes on the FSHR promoter were then mapped using hydroxyl radical footprinting (Tullius et al., 1987). The results from this analysis are shown schematically. One nucleosome positioned very tightly over the region of the promoter from approximately -200 to $-400 \mathrm{bp}$, while the second nucleosome positioned very weakly over the region from -100 to +100 . The $\mathrm{E}$ box appeared to be localized to the linker region and the location of the seven CpG methylation sites $\left(^{*}\right)$ are shown. The model suggests that the upstream nucleosome positions the start site, while the nucleosome over the promoter is much more mobile and can be displaced by demethylation and recruitment of the transcriptional machinery. The methylation or demethylation of the $\mathrm{CpG}$ sites could ultimately determine the ability of cells to express FSHR.

methyl binding proteins and ultimately histone deacetylases and other transcription inactivators. This binding activity could serve to fix the second nucleosome located over the promoter region in place. In addition, methylation of the E box in the linker DNA and perhaps methylation of other sites such as the E2F site could directly prevent binding of a transcription factor. Activation occurs when the DNA is demethylated. Transcription factors could then bind to the DNA and the second nucleosome could be displaced.

Altogether, the data on methylation and chromatin structure support the notion that the transcription of the rat/mouse FSHR gene is regulated by methylation/demethylation of $\mathrm{CpG}$ dinucleotides in the proximal promoter. Although CpG dinucleotides are not abundant in the rat/mouse FSHR promoter, DNA methylation at very specific sites within the promoter correlated with gene inactivation. The maintenance of tissue specificity in gene expression probably requires multiple mechanisms. Our studies support the idea that DNA methylation plays a major role in this process in the regulation of the rat/mouse FSHR gene. It is noteworthy that the proximal $231 \mathrm{bp}$ of the promoter for the human FSHR gene, while $80 \%$ homologous to the promoter for the rat/mouse gene in this region, lacks all of the seven specific $\mathrm{CpG}$ sites and must be regulated by some other mechanism (Gromoll et al., 1996). 


\section{REFERENCES}

Abel MH, Wootton AN, Wilkins V, Huhtaniemi I, Knight PG, Charlton HM 2000 The effect of a null mutation in the follicle-stimulating hormone receptor gene on mouse reproduction. Endocrinology 141(5):1795-1803

Akamizu T, Kosugi S, Kohn LD 1990 Thyrotropin receptor processing and interaction with thyrotropin. Biochem Biophys Res Comm 169(3):947-952

Asa SL, Bamberger AM, Cao B, Wong M, Parker KL, Ezzat S 1996 The transcription activator steroidogenic factor-1 is preferentially expressed in the human pituitary gonadotroph. J Clin Endocrinol Metab 81(6):2165-2170

Barnhart KM, Mellon PL 1994 The orphan nuclear receptor, steroidogenic factor-1, regulates the glycoprotein hormone alpha-subunit gene in pituitary gonadotropes. Mol Endocrinol 8(7): $878-885$

Beard C, Li E, Jaenisch R 1995 Loss of methylation activates Xist in somatic but not in embryonic cells. Genes Dev 9(19):2325-2334

Bender CM, Pao MM, Jones PA 1998 Inhibition of DNA methylation by 5-aza-2'-deoxycytidine suppresses the growth of human tumor cell lines. Cancer Res 58(1):95-101

Bovenzi V, Le NL, Cote S, Sinnett D, Momparler LF, Momparler RL 1999 DNA methylation of retinoic acid receptor beta in breast cancer and possible therapeutic role of 5-aza-2'deoxycytidine. Anticancer Drugs 10(5):471-476

Braun T, Schofield PR, Sprengel R 1991 Amino-terminal leucine rich repeats in gonadotropin receptors determine hormone selectivity. EMBO J 10:1885-1890

Caron KM, Clark BJ, Ikeda Y, Parker KL 1997 Steroidogenic factor 1 acts at all levels of the reproductive axis. Steroids 62(1):53-56

Davy M, Torjesen PA, Aakavaag A 1977 Demonstration of an FSH receptor in a functioning granulosa cell tumour. The effect of gonadotrophin treatment on its viability following transplantation to nude mice. Acta Endocrinol (Copenh) 85(3):615-623

Dierich A, Sairam MR, Monaco L, Fimia GM, Gansmuller A, LeMeur M, Sassone-Corsi P 1998 Impairing follicle-stimulating hormone (FSH) signaling in vivo: targeted disruption of the FSH receptor leads to aberrant gametogenesis and hormonal imbalance. Proc Natl Acad Sci USA 95(23):13612-13617

Dorn C, Ou Q, Svaren J, Crawford PA, Sadovsky Y 1999 Activation of luteinizing hormone beta gene by gonadotropin-releasing hormone requires the synergy of early growth response-1 and steroidogenic factor-1. J Biol Chem 274(20):13870-13876

Duval DL, Nelson SE, Clay CM 1997 A binding site for steroidogenic factor-1 is part of a complex enhancer that mediates expression of the murine gonadotropin-releasing hormone receptor gene. Biol Reprod 56(1):160-168

Eden S, Cedar H 1994 Role of DNA methylation in the regulation of transcription. Curr Opin Genet Dev 4(2):255-259

el-Deiry WS, Nelkin BD, Celano P, Yen RW, Falco JP, Hamilton SR, Baylin SB 1991 High expression of the DNA methyltransferase gene characterizes human neoplastic cells and progression stages of colon cancer. Proc Natl Acad Sci USA 88(8):3470-3474

Fritz KB, Rommerts FG, Louis BG, Dorrington JH 1976 Regulation by FSH and dibutyryl cyclic AMP of the formation of androgen-binding protein in Sertoli cell-enriched cultures. J Reprod Fertil 46:17-24

Goetz TL, Lloyd TL, Griswold MD 1996 Role of E box and initiator region in the expression of the rat follicle-stimulating hormone receptor. J Biol Chem 271(52):33317-33324

Griswold MD 1993 Action of FSH on mammalian Sertoli cells. In: Griswold MD, Russell LD, eds. The Sertoli Cell. Clearwater, FL: Cache River Press; 493-508 
Griswold MD, Kim JS 2001 Site-specific methylation of the promoter alters deoxyribonucleic acidprotein interactions and prevents follicle-stimulating hormone receptor gene transcription. Biol Reprod 64(2):602-610

Griswold MD, Kim J, Tribley WA 2001 Mechanisms involved in the homologous down-regulation of transcription of the follicle-stimulating hormone receptor gene in Sertoli cells. Mol Cell Endocrinol 173(1-2):95-107

Gromoll J, Ried T, Holtgreve-Grez H, Nieschlag E, Gudermann T 1994 Localization of the human FSH receptor to chromosome 2 p21 using a genomic probe comprising exon 10. J Mol Endocrinol 12(3):265-271

Gromoll J, Pekel E, Nieschlag E 1996 The structure and organization of the human folliclestimulating hormone receptor (FSHR) gene. Genomics 35(2):308-311

Gross B, Misrahi M, Sar S, Milgrom E 1991 Composite structure of the human thyrotropin receptor gene. Biochem Biophys Res Comm 177:679-687

Halvorson LM, Ito M, Jameson JL, Chin WW 1998 Steroidogenic factor-1 and early growth response protein 1 act through two composite DNA binding sites to regulate luteinizing hormone beta- subunit gene expression. J Biol Chem 273(24):14712-14720

Halvorson LM, Kaiser UB, Chin WW 1999 The protein kinase C system acts through the early growth response protein 1 to increase LHbeta gene expression in synergy with steroidogenic factor-1. Mol Endocrinol 13(1):106-116

Hamalainen T, Poutanen M, Huhtaniemi I 1999 Age- and sex-specific promoter function of a 2-kilobase 5 '-flanking sequence of the murine luteinizing hormone receptor gene in transgenic mice. Endocrinology 140(11):5322-5329

Hatano O, Takayama K, Imai T, Waterman MR, Takakusu A, Omura T, Morohashi K 1994 Sex-dependent expression of a transcription factor, Ad4BP, regulating steroidogenic P-450 genes in the gonads during prenatal and postnatal rat development. Development 120(10): 2787-2797

Heckert LL 2001 Activation of the rat follicle-stimulating hormone receptor promoter by steroidogenic factor 1 is blocked by protein kinase a and requires upstream stimulatory factor binding to a proximal e box element. Mol Endocrinol 15(5):704-715

Heckert LL, Daley IJ, Griswold MD 1992 Structural organization of the follicle-stimulating hormone receptor gene. Mol Endocrinol 6(1):70-80

Heckert LL, Daggett MA, Chen J 1998 Multiple promoter elements contribute to activity of the follicle-stimulating hormone receptor (FSHR) gene in testicular Sertoli cells. Mol Endocrinol 12(10): 1499-1512

Heckert LL, Sawadogo M, Daggett MA, Chen JK 2000 The USF proteins regulate transcription of the follicle-stimulating hormone receptor but are insufficient for cell-specific expression. Mol Endocrinol 14(11):1836-1848

Hsu SY, Liang SG, Hsueh AJ 1998 Characterization of two LGR genes homologous to gonadotropin and thyrotropin receptors with extracellular leucine-rich repeats and a $\mathrm{G}$ proteincoupled, seven-transmembrane region. Mol Endocrinol 12(12):1830-1845

Hsu SY, Kudo M, Chen T, Nakabayashi K, Bhalla A, van der Spek PJ, van Duin M, Hsueh AJ 2000 The three subfamilies of leucine-rich repeat-containing $\mathrm{G}$ protein-coupled receptors (LGR): identification of LGR6 and LGR7 and the signaling mechanism for LGR7. Mol Endocrinol 14(8):1257-1271

Huhtaniemi IT, Eskola V, Pakarinen P, Matikainen T, Sprengel R 1992 The murine luteinizing hormone and follicle-stimulating hormone receptor genes: transcription initiation sites, putative promoter sequences and promoter activity. Mol Cell Endocrinol 88:55-66

Ikeda Y, Shen WH, Ingraham HA, Parker KL 1994 Developmental expression of mouse steroidogenic factor-1, an essential regulator of the steroid hydroxylases. Mol Endocrinol $8(5): 654-662$ 
Ingraham HA, Lala DS, Ikeda Y, Luo X, Shen WH, Nachtigal MW, Abbud R, Nilson JH, Parker KL 1994 The nuclear receptor steroidogenic factor 1 acts at multiple levels of the reproductive axis. Genes Dev 8(19):2302-2312

Ito M, Park Y, Weck J, Mayo KE, Jameson JL 2000 Synergistic activation of the inhibin alpha-promoter by steroidogenic factor-1 and cyclic adenosine $3{ }^{\prime}, 5^{\prime}$-monophosphate. Mol Endocrinol 14(1):66-81

Jones PL, Veenstra GJ, Wade PA, Vermaak D, Kass SU, Landsberger N, Strouboulis J, Wolffe AP 1998 Methylated DNA and MeCP2 recruit histone deacetylase to repress transcription. Nature Genet 19(2):187-191

Keri RA, Nilson JH 1996 A steroidogenic factor-1 binding site is required for activity of the luteinizing hormone beta subunit promoter in gonadotropes of transgenic mice. J Biol Chem 271(18):10782-10785

Kim JS, Griswold MD 2001 E2F and GATA-1 are required for the Sertoli cell-specific promoter activity of the follicle-stimulating hormone receptor (FSHR) gene. J Androl 22(4):629-639

Kobe B, Deisendorfer J 1994 The leucine-rich repeat: a versatile binding motif. Trends Biochem Sci 19:415-421

Koo YB, Ji I, Slaughter RG, Ji TH 1991 Structure of the leuteinizing hormone receptor gene and multiple exons of the coding sequence. Endocrinology 128:2297-2650

Krishnamurthy H, Danilovich N, Morales CR, Sairam MR 2000 Qualitative and quantitative decline in spermatogenesis of the follicle-stimulating hormone receptor knockout (FORKO) mouse. Biol Reprod 62(5):1146-1159

Kumar TR, Wang Y, Lu N, Matzuk M 1997 Follicle stimulating hormone is required for ovarian follicle maturation but not male fertility. Nature Genet 15:201-204

Levallet J, Koskimies P, Rahman N, Huhtaniemi I 2001 The promoter of murine folliclestimulating hormone receptor: functional characterization and regulation by transcription factor steroidogenic factor 1. Mol Endocrinol 15(1):80-92

Linder CC, Heckert LL, Goetz TL, Griswold MD 1994 Follicle-stimulating hormone receptor gene promoter activity. Endocr J 2:957-966

Loosfelt H, Misrahi M, Atger M, Salesse R, Thi MTVH-L, Jolivet A, Guiochon-Mantel A, Sar S, Jallal B, Garnier J, Milgrom E 1989 Cloning and sequencing of porcine LH-hCG receptor cDNA: variants lacking transmembrane domain. Science 245:525-528

Maguire SM, Tribley WA, Griswold MD 1997 Follicle-stimulating hormone (FSH) regulates the expression of FSH receptor messenger ribonucleic acid in cultured Sertoli cells and in hypophysectomized rat testis. Biol Reprod 56(5):1106-1111

McFarland KC, Sprengel R, Phillips HS, Köhler M, Rosemblit N, Nikolics K, Segaloff DL, Seeburg PH 1989 Lutropin-choriogonadotropin receptor: an unusual member of the G protein-coupled receptor family. Science 245:494-499

McGuinness MP, Linder CC, Morales CR, Heckert LL, Pikus J, Griswold MD 1994 Relationship of a mouse Sertoli cell line (MSC-1) to normal Sertoli cells. Biol Reprod 51(1):116-124

Meachem SJ, McLachlan R, de Kretser D, Robertson DM, Wreford NG 1996 Neonatal exposure of rats to recombinant follicle stimulating hormone increases adult Sertoli cell and spermatogenic cell numbers. Biol Reprod 54:36-44

Means AR, Fakunding JL, Huckins C, Tindall DJ, Vitale R 1976 Follicle-stimulating hormone, the Sertoli cell, and spermatogenesis. Recent Progr Horm Res 32(477):477-527

Monaco L, Foulkes NS, Sassone-Corsi P 1995 Pituitary follicle-stimulating hormone (FSH) induces CREM gene expression in Sertoli cells: involvement in long-term desensitization of the FSH receptor. Proc Natl Acad Sci USA 92(23):10673-10677

Morohashi K, Honda S, Inomata Y, Handa H, Omura T 1992 A common trans-acting factor, Ad4-binding protein, to the promoters of steroidogenic P-450s. J Biol Chem 267(25):1791317919 
Morohashi K, Hatano O, Nomura M, Takayama K, Hara M, Yoshii H, Takakusu A, Omura T 1995 Function and distribution of a steroidogenic cell-specific transcription factor, Ad4BP. J Steroid Biochem Mol Biol 53(1-6):81-88

Nan X, Ng HH, Johnson CA, Laherty CD, Turner BM, Eisenman RN, Bird A 1998 Transcriptional repression by the methyl-CpG-binding protein MeCP2 involves a histone deacetylase complex. Nature 393(6683):386-389

Ngan ES, Cheng PK, Leung PC, Chow BK 1999 Steroidogenic factor-1 interacts with a gonadotrope-specific element within the first exon of the human gonadotropin-releasing hormone receptor gene to mediate gonadotrope-specific expression. Endocrinology 140(6): 2452-2462

Nickel J, Short ML, Schmitz A, Eggert M, Renkawitz R 1995 Methylation of the mouse M-lysozyme downstream enhancer inhibits heterotetrameric GABP binding. Nucleic Acids Res 23(23):4785-4792

Orth J 1984 The role of follicle-stimulating hormone in controlling Sertoli cells proliferation in testes of fetal rats. Endocrinology 115:1248-1255

Orth JM, Gunsalus GL, Lamperti AA 1988 Evidence from Sertoli cell-depleted rats indicates that spermatid number in adults depends on numbers of Sertoli cells produced during perinatal development. Endocrinology 122(3):787-794

Ottaviano YL, Issa JP, Parl FF, Smith HS, Baylin SB, Davidson NE 1994 Methylation of the estrogen receptor gene $\mathrm{CpG}$ island marks loss of estrogen receptor expression in human breast cancer cells. Cancer Res 54(10):2552-2555

Parmentiar M 1989 Molecular cloning of the thyrotropin receptor. Science 246:1620-1622

Reeves R, Wolffe A 1996 Substrate structure influences binding of the non-histone protein HMI-Y to free and nucleosomal DNA. Biochemistry 35:5063-5074

Rice DA, Mouw AR, Bogerd AM, Parker KL 1991 A shared promoter element regulates the expression of three steroidogenic enzymes. Mol Endocrinol 5(10):1552-1561

Richardson B, Yung R 1999 Role of DNA methylation in the regulation of cell function. J Lab Clin Med 134(4):333-340

Roselli CE, Jorgensen EZ, Doyle MW, Ronnekleiv OK 1997 Expression of the orphan receptor steroidogenic factor-1 mRNA in the rat medial basal hypothalamus. Brain Res Mol Brain Res 44(1):66-72

Sairam MR, Subbarayan VS 1997 Characterization of the $5^{\prime}$ flanking region and potential control elements of the ovine follitropin receptor gene. Mol Reprod Dev 48(4):480-487

Shen WH, Moore CC, Ikeda Y, Parker KL, Ingraham HA 1994 Nuclear receptor steroidogenic factor 1 regulates the mullerian inhibiting substance gene: a link to the sex determination cascade. Cell 77(5):651-661

Singh J, Handelsman DJ 1996 Neonatal administration of FSH increases Sertoli cell numbers and spermatogenesis in gonadotropin-deficient $(h p g)$ mice. J Endocrinol 151:37-48

Singh J, O'Neill C, Handelsman DJ 1995 Induction of spermatogenesis by androgens in gonadotropin-deficient ( $h p g$ ) mice. Endocrinology 136(12):5311-5321

Smale S, Baltimore D 1989 The "initiator" as a transcription control element. Cell 57:103-113

Sprengel R, Braun T, Nikolics K, Segaloff D, Seeburg P 1990 The testicular receptor for follicle stimulating hormone: structure and functional expression of cloned cDNA. Mol Endocrinol 4:525-530

Tapanainen JS, Aittomaki K, Min J, Vaskivuo T, Huhtaniemi IT 1997 Men homozygous for an inactivating mutation of the follicle-stimulating hormone (FSH) receptor gene present variable suppression of spermatogenesis and fertility. Nature Genet 15(2):205-206

Tate PH, Bird AP 1993 Effects of DNA methylation on DNA-binding proteins and gene expression. Curr Opin Genet Dev 3(2):226-231 
Themmen A, Blok L, Post M, Baarends W, Hoogerbrugge J, Parmentier M, Vassart G, Grootegood A 1991 Follitropin receptor down regulation involves a cAMP-dependent post-transcriptional decrease of receptor mRNA expression. Mol Cell Endocrinol 78:R7-R13

Tremblay JJ, Drouin J 1999 Egr-1 is a downstream effector of GnRH and synergizes by direct interaction with Ptx1 and SF-1 to enhance luteinizing hormone beta gene transcription. Mol Cell Biol 19(4):2567-2576

Tsai-Morris C, Buczko E, Wang W, Xie X, Dufau M 1991 Structural organization of the rat leutinizing hormone receptor gene. J Biol Chem 266:11,355-11,359

Tullius TD, Dombroski BA, Churchill MEA, Kam L 1987 Hydroxyl radical footprinting: a high resolution method for mapping protein-DNA contacts. Meth Enzymol 155:537-558

Vibede N, Hauser F, Williamson M, Grimmelikhuijzen CJ 1998 Genomic organization of a receptor from sea anemones, structurally and evolutionarily related to glycoprotein hormone receptors from mammals. Biochem Biophys Res Commun 252(2):497-501

Weis L, Reinberg D 1992 Transcription by RNA polymerase II: initiator-directed formation of transcription-competent complexes. FASEB J 6(14):3300-3309

Wolfe MW 1999 The equine luteinizing hormone beta-subunit promoter contains two functional steroidogenic factor-1 response elements. Mol Endocrinol 13(9):1497-1510

Wolfe MW, Call GB 1999 Early growth response protein 1 binds to the luteinizing hormone-beta promoter and mediates gonadotropin-releasing hormone-stimulated gene expression. Mol Endocrinol 13(5):752-763

Xing W, Sairam MR 2001 Characterization of regulatory elements of ovine follicle-stimulating hormone (FSH) receptor gene: the role of E-box in the regulation of ovine FSHreceptor expression. Biol Reprod 64(2):579-589

Zirkin BR, Awoniyi C, Griswold MD, Russell LD, Sharpe R 1994 Is FSH required for adult spermatogenesis? J Androl 15(4):273-276 